\title{
Investigation of the Pathogenesis and Treatment Efficiency of Bevacizumab-Induced Hypertension in the Rat Model
}

\author{
*Mehmet Ali Balcı' ${ }^{1}$, Musa Özgür Özyiğit'², Volkan İpek², İlker Mustafa Kafa ${ }^{3}$, \\ Ender Kurt ${ }^{4}$
}

\author{
${ }^{1}$ Uludag University, School of Medicine, Department of İnternal Medicine, \\ ${ }^{2}$ Uludag University, Faculty of Veterinary Medicine, Department of Pathology, \\ ${ }^{3}$ Uludag University, School of Medicine, Department of Anatomy, \\ ${ }^{4}$ Uludag University, School of Medicine, Department of Oncology, \\ Bursa Turkey. *E-mail: abalci13@gmail.com \\ DOI: $10.31964 / \mathrm{mltj} . v 5 i 1.209$
}

\begin{abstract}
Bevacizumab is known to reduce Vascular Endothelial Growth Factor (VEGF) to undetectable levels when used in conjunction with chemotherapy. Hypertension is a frequent adverse effect of bevacizumab, although its mechanism(s) remain unclear. In this study, our aim was to examine the pathogenesis of bevacizumab-induced hypertension and to investigate the treatment efficacy of valsartan. A total of 24 Wistar Albino female rats were included in the study. Rats were divided into three groups with 8 rats in each, as follows: The control group, bevacizumab group and bevacizumab + valsartan group. Blood pressure, blood urea nitrogen and serum creatinine levels were measured, urine samples were collected for 24 hours statistical analyses were performed using IBM SPSS 20 software pack. Nephrectomy specimens in bevacizumab and bevacizumab + valsartan groups exhibited varying degrees of renal injury. Although valsartan was able to reduce the bevacizumab-induced rise in blood pressure, it could not prevent the development of nephropathy. Conclusions these findings suggest that hypertension occurring secondary to bevacizumab treatment in rats may be associated with mechanisms involving renal injury.
\end{abstract}

Keywords: Bevacizumab; hypertension; valsartan; rat model

\section{INTRODUCTION}

Since vascular endothelial growth factor (VEGF) mediated tumor angiogenesis plays a significant role in tumor growth, dissemination and metastasis, anti-VEGF treatment have become the main focus in cancer treatment (Folkman, 2002; Hicklin \& Ellis, 2005). Bevacizumab, a recombinant human Vascular Endothelial Growth Factor-A monoclonal antibody (anti-VEGF-A) is known to reduce serum VEGF to undetectable levels while inhibiting the growth of several tumors (Hurwitz et al., 2004).

Hypertension is a common undesired side effect of bevacizumab therapy, and many theories have been put forward to explain hypertension associated with VEGF inhibitor treatment (Figure 1) (Touyz, Lang, Herrmann, van den Meiracker, \& Danser, 2017). These include the nitric oxide theory, peripheral vascular resistance theory, renal failure theory, and pre-eclampsia theory (Syrigos, Karapanagiotou, Boura, Manegold, \& Harrington, 2011). In another study, it was concluded that genetic variation in WNK1, KLKB1, and GRK4 might be associated with Bevacizumab 
induced hypertension (Frey et al., 2017). Administration of anti-VEGFR2 Semaxanib (SU5416) in rats resulted in renal histological abnormalities and increased arterial pressure (Gu et al., 2009). Inhibition of VEGF-mediated vasodilation is the dominant hypothesis for the pathophysiology of bevacizumab-induced hypertension ( $\mathrm{Li}$ \& Kroetz, 2018). Previously; bevacizumab-associated hypertension model not studied on rats.

In this study, we aimed to examine the pathogenesis of bevacizumab-induced hypertension and to investigate the treatment efficacy of valsartan.

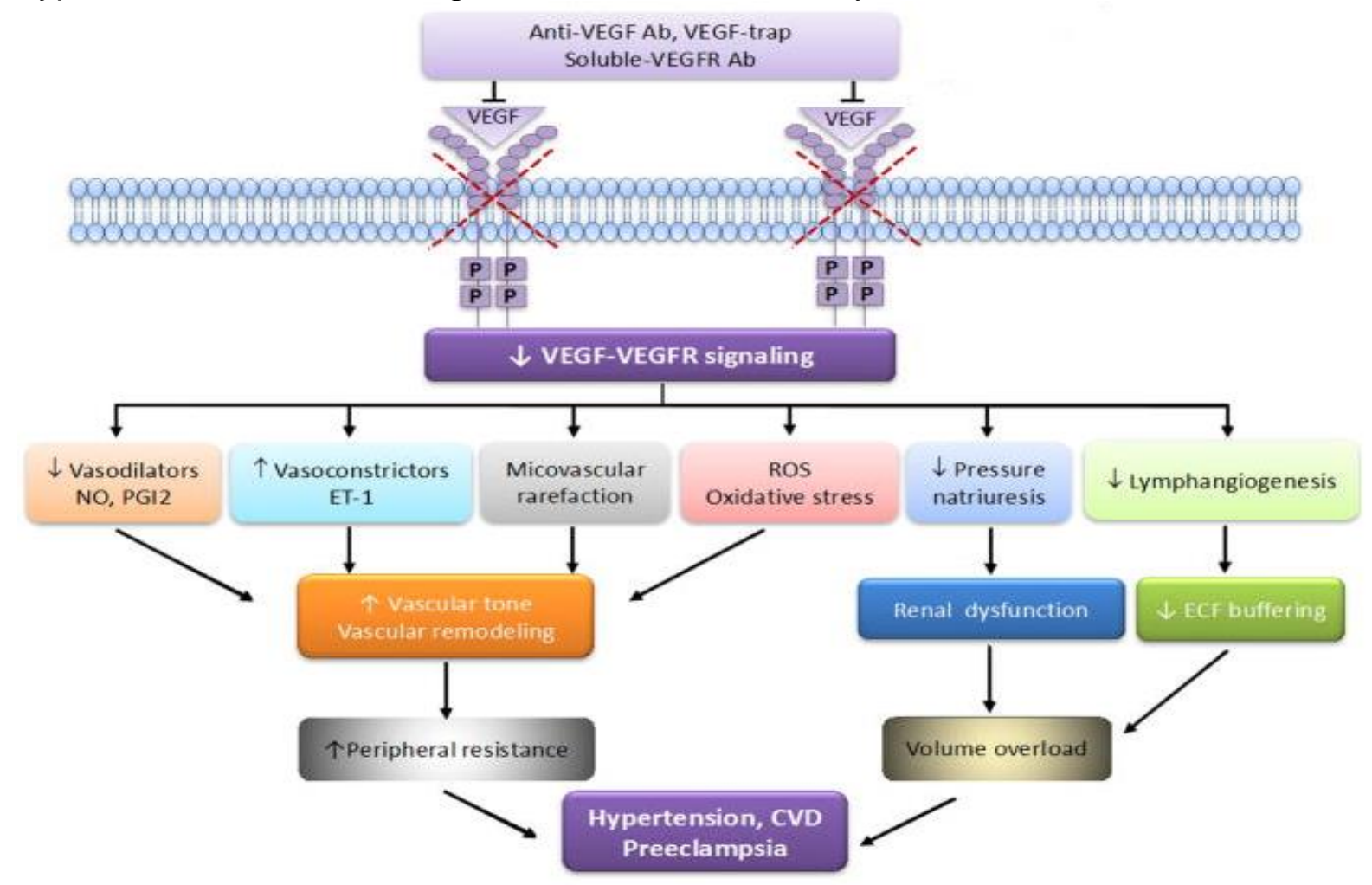

Figure 1: Theories about VEGF-VEGFR inhibition Induced Hypertension. These processes result in reduced VEGFR signaling and consequent reduction in the production of vasodilators ( $\mathrm{NO}$ and $\mathrm{PGI} 2$ ), increased production of vasoconstrictors (ET-1), oxidative stress and rarefaction, resulting in increased vascular tone and arterial remodeling. Reduced pressure natriuresis and impaired lymphatic function contribute to volume overload. $p$, phosphorylation site of tyrosine kinase; ROS, reactive oxygen species; NO, nitric oxide; ET-1, endothelin-1; Ab, antibody; ECF, extracellular fluid; CVD, cardiovascular disease, PGI2, prostaglandin I2. (Modified from Touyz RM, Lang NN, Herrmann J, van den Meiracker AH, Danser AHJ. Recent Advances in Hypertension and Cardiovascular toxicities with VEGF Inhibition. Hypertension. 2017; 70:220-226).

\section{MATERIALS AND METHODS}

A total of 24 female Wistar Albino rats aged between 2 and three months and weighing $200 \mathrm{~g}$ included in the study. All rats belonged to the same generation, and were maintained and fed under room temperature conditions $\left(22^{\circ} \mathrm{C}\right)$. Animal experimentation performed by the national guidelines for use and care of laboratory animals, and the study protocol was approved by the Local Ethics Committee for Experimental Animals, Uludağ University. 


\section{Experimental Groups}

Group $1(n=8)$ : Control group. The rats in this group received physiological saline intraperitoneally at a dose of $10 \mathrm{ml} / \mathrm{kg} /$ week. Group $2(\mathrm{n}=8)$ : The rats in this group received bevacizumab intraperitoneally at a dose of $10 \mathrm{mg} / \mathrm{kg} / \mathrm{week}$. Group 3 $(n=8)$ : The rats in this group received oral valsartan via the drinking water at a dose of $10 \mathrm{mg} / \mathrm{kg} / \mathrm{day}$ plus $10 \mathrm{mg} / \mathrm{kg} /$ week bevacizumab intraperitoneally.

\section{Experimental Protocol}

Blood pressure measurements performed at ten weeks of study in all experimental animals. After the study, intracardiac blood samples obtained, and nephrectomy specimens assessed by a single pathologist.

\section{Blood Pressure Measurements}

Blood Pressure (BPs) values were determined using the indirect tail-cuff method (MAY BPHR 9610-PC Tail-Cuff Indirect BP Recorder; Comment Ltd., Ankara, Turkey). Each cage contained only one rat. Measurements were performed in a quiet and calm laboratory environment and recorded digitally

\section{Biochemistry index assay}

Blood urea nitrogen and serum creatinine levels were measured using picric acid creatinine reagent.

\section{Urine collection}

Urine samples collected for 24 hours, from 8.00 am to $8.00 \mathrm{pm}$ next day at Week 0 (before injection), 3, 6, 9, and 12. Measurements were performed using turbidimetric methods (Cobas Integra 800).

\section{Surgical Preparation}

Rats sedated with ketalar + rompun at the end of the 12th week. A midline incision made, and blood samples obtained from the left ventricle. Both kidneys of each rat were totally excised.

\section{Pathological Assessments}

Tissue samples for pathological assessments fixed for $24 \mathrm{~h}$ in formaldehyde solution with $10 \%$ neutral buffer. After routine tissue processing, four micron-thick cross-sections prepared from paraffin blocks stained with hematoxylin and eosin. Samples were histologically graded under light microscopic examination, using the methodology described by Bayar et al. (Bayar MK, 1998). As follows: 0, no pathology; 1, mild injury consisting of glomerular mesangial proliferation, interstitial vascular congestion; 2 , moderate damage with thickening of the basal membrane, accumulation of substance in the tubules, interstitial nephritis; 3 , severe injury with fatty degeneration of the cellular structures and permanent changes in the basal layer.

\section{Statistical Analyses}

Statistical analyses performed at the Department of Biostatistics, Uludağ University using IBM SPSS 20 software pack. The normal distribution of the data tested with the Shapiro Wilk test. For continuous variables, median (minimummaximum) values were provided as descriptive statistics. In all three groups (control group, bevacizumab group, and bevacizumab + valsartan group) analyses were 
performed for blood pressure values at ten weeks, blood sample results after the experiment, and pathological assessments. The significance of the differences between the three groups examined with the Kruskal Wallis test. If a significant difference found, pairwise comparisons using the Mann-Whitney $U$ test performed. For each group, the correlations between pathological grade and blood pressure were investigated using Spearmen's correlation test, where a correlation coefficient of $0.2-0.4,0.4-0.7$, and $>0.7$ considered as mild, moderate, and strong correlation, respectively - the significance set at an alpha level of $<0.05$.

\section{RESULTS AND DISCUSSION}

Mean systolic and diastolic blood pressures were higher in bevacizumab group as compared to control $(p>0.05)$ and bevacizumab + valsartan groups $(p=0.08)$. On the other hand, mean systolic and diastolic blood pressure values were lower in bevacizumab + valsartan group than in bevacizumab $(p>0.05)$ and control $(p>0.05)$ groups $(p>0.05)$. Table 1 shows a more detailed summary of blood pressure measurements.

Table 1. Blood pressure measurements.

\begin{tabular}{lccc}
\hline & Control & Bevacizumab & Bevacizumab+Valsartan \\
\hline Systolic BP & $130.4(108.6-$ & $146.1(89-242.7)$ & $110(91.6-143.6)$ \\
& $165.7)$ & & \\
Diastolic & $94.3(70.6-113.4)$ & $110.7(63.5-$ & $81.7(39.9-105.4)$ \\
BP & & $197.8)$ & \\
\hline
\end{tabular}

BP: blood pressure

Biochemical tests

Kidney function tests and electrolyte levels were comparable across all three study groups (Table 2).

Table 2. Results of biochemical analyses

\begin{tabular}{lccc}
\hline & Control & Bevacizumab & $\begin{array}{c}\text { Bevacizumab+ } \\
\text { Valsartan }\end{array}$ \\
\hline Urea, $\mathbf{m g} / \mathbf{d L}$ & $48.25(35.8-65.1)$ & $47.75(36.8-58.2)$ & $45.4(38.5-48.4)$ \\
Creatinine, $\mathbf{m g} / \mathbf{d L}$ & $0.34(0.31-0.82)$ & $0.28(0.23-0.33)$ & $0.31(0.26-0.41)$ \\
Sodium, $\mathbf{m E q} / \mathbf{L}$ & $138.75(134.6-$ & $139.6(130.8-$ & $136.0(129.8-$ \\
& $140.8)$ & $41.0)$ & $142.0)$ \\
Potassium, $\mathbf{m E q} / \mathbf{L}$ & $5.7(4.5-8.3)$ & $5.3(4.3-7.4)$ & $5.5(4.6-13.3)$ \\
Calcium, $\mathbf{m g} / \mathbf{d L}$ & $10.6(6.1-11.5)$ & $10.9(9.9-11.4)$ & $11.2(9.8-13.1)$ \\
\hline
\end{tabular}

\section{4-hour Proteinuria ( $\mathrm{mg} / \mathrm{dL}$ )}

In all groups, 24-hour urinary protein analyses were standard, with no significant differences between the study groups at $0,3,6,9$, and 12 weeks (Figure 2). A moderate, but statistically insignificant positive correlation between the histological grade and proteinuria noted in the bevacizumab group ( $\mathrm{r} s=0.682$, $\mathrm{p}=0.063$ ). 


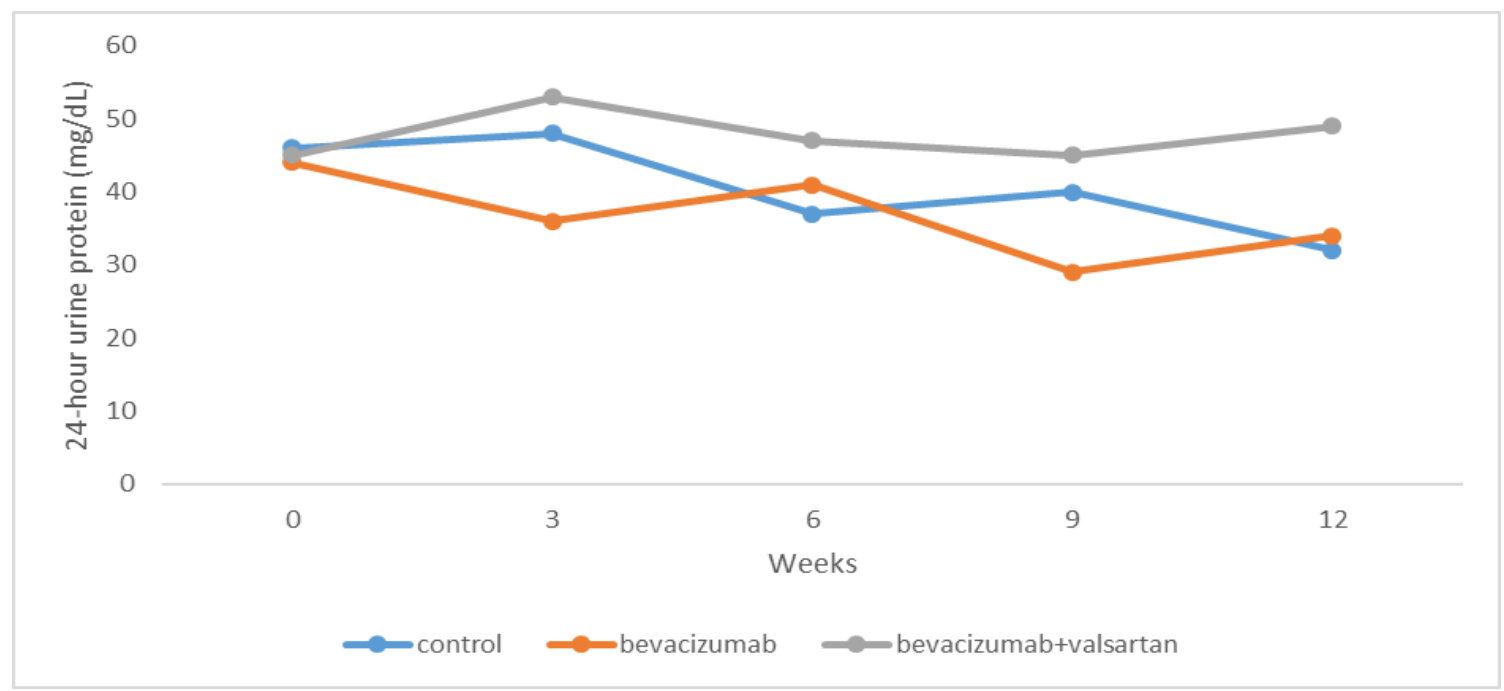

Figure 2: 24-hour urinary protein measurements in study groups

\section{Histopathological findings}

Histopathological grades were similar in bevacizumab and bevacizumab + valsartan groups (median 2, range 1-3 for both groups). Histological grade was 0 in all the rats in the control group, while it was significantly higher in bevacizumab and bevacizumab + valsartan groups compared to controls $(p<0.001$ for both comparisons). Grade-0, grade-1, grade-2, grade-3 nephropathy samples shown in Figure 3.

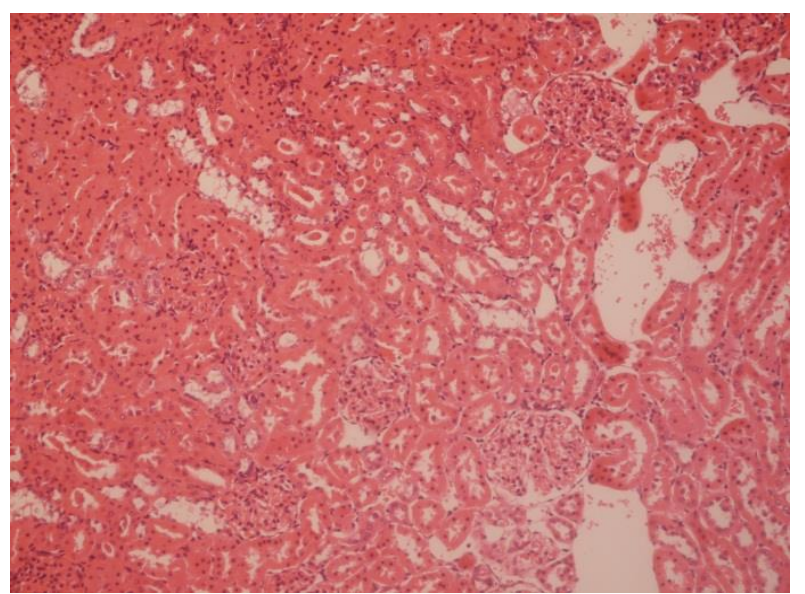

A: Normal view Grade: $0, \mathrm{H}$ \& E 10X magnification.

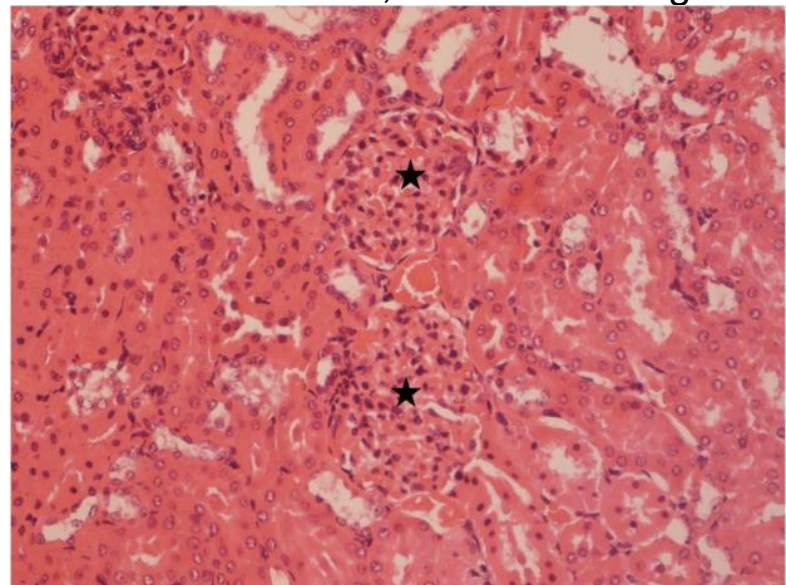

B: Grade: 1, Glomerular hypercellularity (stars), H \& E 20X magnification 


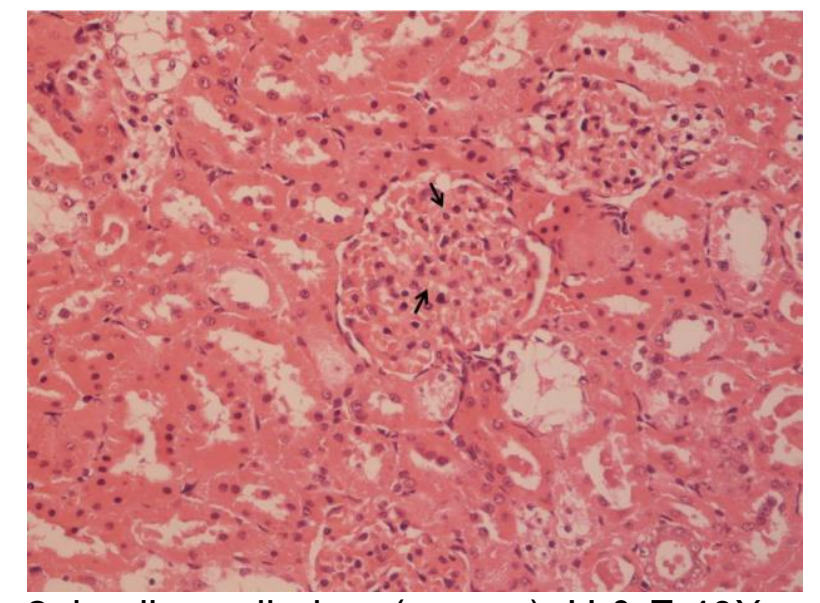

C: Grade: 2, hyaline cylinders (arrows), H \& E 40X magnification

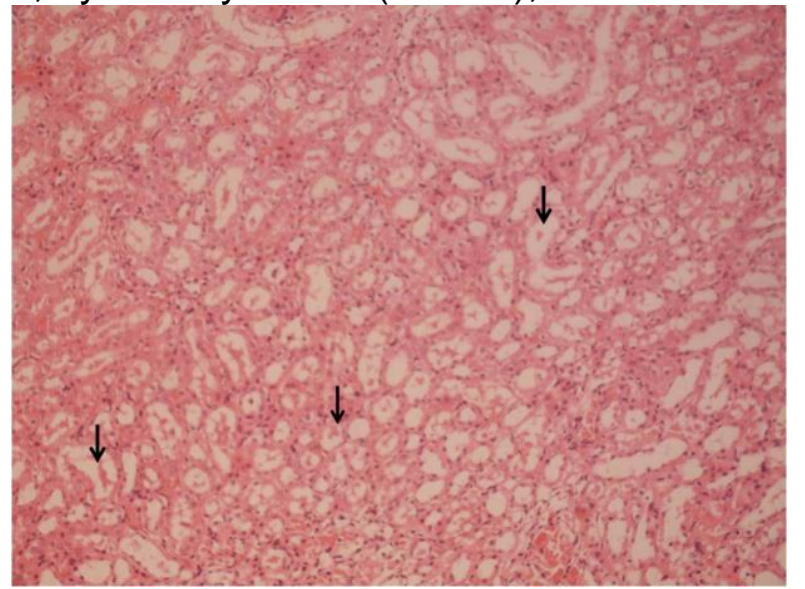

D: Grade: 3, increased glomerular mesangium (arrows), H \& E 10X magnification

Figure 3. Examples for histological grades.

In this study, rats in the bevacizumab group had higher mean blood pressure values as compared to other groups, while bevacizumab + valsartan group had lower mean blood pressure as compared to controls. Assessment of 24-hour protein showed no difference between the groups. In the bevacizumab group, the histopathological grade at 12 weeks showed a moderately positive, statistically insignificant association with proteinuria.

Recent studies show an increased incidence of hypertension and proteinuria in patients receiving anti-VEGF antibody agents. In a systematic review and comprehensive meta-analysis of 72 published studies, the prevalence of hypertension and proteinuria in patients receiving bevacizumab were $25.3 \%(95 \% \mathrm{Cl}$ : $21.5 \%-29.5 \%$ ) and $18 \%$ (95\% Cl: 11.5\%-26.6\%), respectively (Zhao, Wang, Xu, Xu, \& Liu, 2017). In another study, renal disease induced by anti-VEGF infusion was characterized by nephrotic proteinuria, endotheliosis, glomerulosclerosis, and hyaline deposition, as shown by renal biopsy examinations (Izzedine, Rixe, Billemont, Baumelou, \& Deray, 2007). In this regard, it is interesting to note that pregnancies complicated by preeclampsia are associated with elevated soluble VEGF receptor one protein (sFlt-1), endothelial cell dysfunction, and proteinuria. Such observations suggest that neutralization of physiologic levels of VEGF, a critical endothelial survival factor, may lead to proteinuria (Sugimoto et al., 2003). The reduction of VEGFA production by the podocytes leads to loss of glomerular cells, resulting in mesangiolysis. Bevacizumab-associated proteinuria may partly be a consequence of increased intraglomerular pressure caused by hypertension. Despite an association 
between hypertension and proteinuria, it is a challenging task to determine whether proteinuria caused by hypertension or hypertension is secondary to renal damage. It is also possible that both hypertension and proteinuria independently generated by VEGF blockage.

Some studies have reported biopsy findings associated with bevacizumab induced renal damage. For instance, one patient in the low-dose arm of the trial by Johnson et al. (Johnson et al., 2004) developed clinically apparent nephrotic syndrome. This patient's biopsy showed the presence of cryoglobulinemic glomerulonephritis. In the study by Miller et al., (Miller et al., 2005) in one patient developing nephrotic syndrome, renal biopsy showed collapsing glomerulopathy. Also, the results of the histopathological examination reported in a patient who developed new-onset hypertension and nephrotic syndrome in association with bevacizumab treatment for metastatic pancreatic cancer. This biopsy showed immune-complex-mediated focal proliferative glomerulonephritis. Nephrotic syndrome and hypertension resolved after discontinuation of bevacizumab (George, Zhou, \& Toto, 2007).

In our study, histopathological examination of the nephrectomy specimens showed varying degrees of glomerular mesangial proliferation, interstitial vascular congestion, thickening of the basal membrane, accumulation of substance in the tubules, interstitial nephritis, fatty degeneration of the cellular structures, and permanent changes in the basal layer in rats treated with bevacizumab or bevacizumab + valsartan. A significant difference in histological grade found between control rats and the rats in the other two groups. Despite lower BP measurements in the bevacizumab + valsartan group, the degree of the kidney injury was similar between bevacizumab + valsartan and bevacizumab groups.

Limitations of our study include a lack of NO, VEGF, and sFlt-1 as well as basal blood pressure measurements.

\section{CONCLUSION}

Our results suggest that hypertension occurring secondary to bevacizumab treatment in rats may be associated with mechanisms involving renal injury. Studies involving simultaneous measurements of nitric oxide, VEGF, and sFIt-1 may provide further insights into the mechanisms of bevacizumab-induced hypertension and renal damage.

\section{REFERENCES}

Bayar MK, Ö. E., Özercan İ, Erhan ÖL. (1998). Tavşanlarda tekrarlanan dozlarda kullanılan sevofluranın oluşturduğu renal histopatolojik değişiklikler ve plazma florür düzeyine etkileri. Anestezi Dergisi, 6, 144.

Folkman, J. (2002). Role of angiogenesis in tumor growth and metastasis. Semin Oncol, 29(6 Suppl 16), 15-18. doi:10.1053/sonc.2002.37263

Frey, M. K., Dao, F., Olvera, N., Konner, J. A., Dickler, M. N., \& Levine, D. A. (2017). Genetic predisposition to bevacizumab-induced hypertension. Gynecol Oncol, 147(3), 621-625. doi:10.1016/j.ygyno.2017.09.017

George, B. A., Zhou, X. J., \& Toto, R. (2007). Nephrotic syndrome after bevacizumab: case report and literature review. Am J Kidney Dis, 49(2), e2329. doi:10.1053/j.ajkd.2006.11.024

Gu, J. W., Manning, R. D., Jr., Young, E., Shparago, M., Sartin, B., \& Bailey, A. P. (2009). Vascular endothelial growth factor receptor inhibitor enhances dietary 
salt-induced hypertension in Sprague-Dawley rats. Am J Physiol Regul Integr Comp Physiol, 297(1), R142-148. doi:10.1152/ajpregu.90972.2008

Hicklin, D. J., \& Ellis, L. M. (2005). Role of the vascular endothelial growth factor pathway in tumor growth and angiogenesis. J Clin Oncol, 23(5), 1011-1027. doi:10.1200/JCO.2005.06.081

Hurwitz, H., Fehrenbacher, L., Novotny, W., Cartwright, T., Hainsworth, J., Heim, W., . . . Kabbinavar, F. (2004). Bevacizumab plus irinotecan, fluorouracil, and leucovorin for metastatic colorectal cancer. N Engl J Med, 350(23), 23352342. doi:10.1056/NEJMoa032691

Izzedine, H., Rixe, O., Billemont, B., Baumelou, A., \& Deray, G. (2007). Angiogenesis inhibitor therapies: focus on kidney toxicity and hypertension. Am J Kidney Dis, 50(2), 203-218. doi:10.1053/j.ajkd.2007.04.025

Johnson, D. H., Fehrenbacher, L., Novotny, W. F., Herbst, R. S., Nemunaitis, J. J., Jablons, D. M., . . . Kabbinavar, F. (2004). Randomized phase II trial comparing bevacizumab plus carboplatin and paclitaxel with carboplatin and paclitaxel alone in previously untreated locally advanced or metastatic nonsmall-cell lung cancer. $J$ Clin Oncol, 22(11), 2184-2191. doi:10.1200/JCO.2004.11.022

Li, M., \& Kroetz, D. L. (2018). Bevacizumab-induced hypertension: Clinical presentation and molecular understanding. Pharmacol Ther, 182, 152-160. doi:10.1016/j.pharmthera.2017.08.012

Miller, K. D., Chap, L. I., Holmes, F. A., Cobleigh, M. A., Marcom, P. K., Fehrenbacher, L., . . . Rugo, H. S. (2005). Randomized phase III trial of capecitabine compared with bevacizumab plus capecitabine in patients with previously treated metastatic breast cancer. J Clin Oncol, 23(4), 792-799. doi:10.1200/JCO.2005.05.098

Sugimoto, H., Hamano, Y., Charytan, D., Cosgrove, D., Kieran, M., Sudhakar, A., \& Kalluri, R. (2003). Neutralization of circulating vascular endothelial growth factor (VEGF) by anti-VEGF antibodies and soluble VEGF receptor 1 (sFlt-1) induces proteinuria. $J$ Biol Chem, 278(15), 12605-12608. doi:10.1074/jbc.C300012200

Syrigos, K. N., Karapanagiotou, E., Boura, P., Manegold, C., \& Harrington, K. (2011). Bevacizumab-induced hypertension: pathogenesis and management. BioDrugs, 25(3), 159-169. doi:10.2165/11590180-000000000-00000

Touyz, R. M., Lang, N. N., Herrmann, J., van den Meiracker, A. H., \& Danser, A. H. J. (2017). Recent Advances in Hypertension and Cardiovascular Toxicities With Vascular Endothelial Growth Factor Inhibition. Hypertension, 70(2), 220-226. doi:10.1161/HYPERTENSIONAHA.117.08856

Zhao, T., Wang, X., Xu, T., Xu, X., \& Liu, Z. (2017). Bevacizumab significantly increases the risks of hypertension and proteinuria in cancer patients: $A$ systematic review and comprehensive meta-analysis. Oncotarget, 8(31), 51492-51506. doi:10.18632/oncotarget.18190 